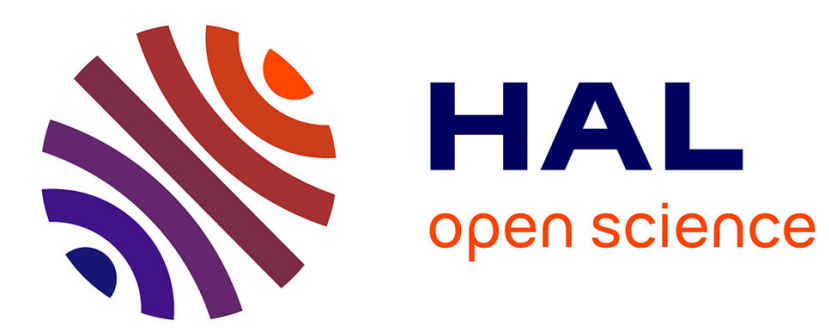

\title{
La fonction des modèles dans l'articulation chronologique du Brutus
}

Jean-Michel David

\section{To cite this version:}

Jean-Michel David. La fonction des modèles dans l'articulation chronologique du Brutus. Le Brutus de Cicéron, Rhétorique, politique et histoire culturelle, 2008, Paris, France. pp.19-38, 10.1163/9789004278738_003. hal-01139363

\section{HAL Id: hal-01139363 https://hal.science/hal-01139363}

Submitted on 4 Apr 2015

HAL is a multi-disciplinary open access archive for the deposit and dissemination of scientific research documents, whether they are published or not. The documents may come from teaching and research institutions in France or abroad, or from public or private research centers.
L'archive ouverte pluridisciplinaire HAL, est destinée au dépôt et à la diffusion de documents scientifiques de niveau recherche, publiés ou non, émanant des établissements d'enseignement et de recherche français ou étrangers, des laboratoires publics ou privés. 


\section{La fonction des modèles dans l'articulation chronologique du Brutus}

Comme Cicéron l'indiquait clairement, son objectif était avec le Brutus de tracer une histoire de l'éloquence romaine'. À la différence de bien d'autres, le dialogue était placé au temps présent. En procédant ainsi, Cicéron renonçait à faire référence à une situation idéale qui lui aurait été fournie par le recours à des personnages dont l'autorité était enracinée dans le passé. Il saisissait au contraire l'occasion de la mort récente d'Hortensius ${ }^{2}$ pour se mettre en scène, accompagné de deux de ses proches amis, Atticus et $\mathrm{M}$. Iunius Brutus, et déplorer le triste état où était parvenu l'art auquel il avait consacré toute sa vie :

[...] hunc autem aut praeter ceteros aut cum paucis sustineret dolorem, cum forum populi Romani, quod fuisset quasi theatrum illius ingeni, uoce erudita et Romanis Graecisque auribus digna spoliatum atque orbatum nideret ${ }^{3}$.

[...] mais il est une douleur dont, plus que tous les autres, ou tout au moins avec peu d'autres personnes, il aurait à porter le poids, ce serait de voir le forum du peuple romain, ce forum qui avait été comme le théâtre de son beau génie, dépouillé et déshérité des accents de cette voix savante, digne des oreilles latines et même des grecques.

Pourtant, ce n'était pas un déclin que ce dialogue retraçait mais bien une progression qui avait conduit à une apogée que Cicéron, sans le dire trop ouvertement, estimait représenter. Il y avait donc là un paradoxe. Comment la chute pouvait-elle être si proche du sommet ? Pourquoi représenter une telle ascension, si c'était pour souligner une telle déchéance ${ }^{4}$ ? [19]

La raison était politique. La domination que César imposait à Rome, contraignait l'éloquence au silence. Sa crise était une crise de la cité. Il ne faut pas en effet perdre de vue que, pour Cicéron, sa fonction essentielle était de permettre l'expression et le débat entre citoyens et donc de créer les conditions d'existence de la communauté civiques. Son histoire était ainsi une histoire politique qui s'inscrivait dans le fonctionnement de la République et en subissait toutes les vicissitudes. Elle se coulait dans ses règles, grandissait quand les débats qui l'animaient se faisaient intenses, et diminuait quand une domination monarchique venait les étouffer.

Un des traits du dialogue qui permet de le confirmer tient à son organisation. Comme toute histoire, elle était chronologique. Dans le cas du Brutus cependant, elle se construisait dans la succession des orateurs qui l'avaient faite : de oratoribus quando esse coepissent, qui etiam et

\footnotetext{
${ }^{1}$ Brut. 20.

${ }^{2}$ Le dialogue a été composé au printemps 46 . Il est supposé se dérouler au même moment. La mort d'Hortensius à laquelle Cicéron fait allusion (§ 1-7) remonte au printemps 50. Cf. sur ce point infra, p. 216, les remarques de P. M. Martin.

${ }^{3}$ Brut. 6 ; trad. J. Martha. Cf. Brut. 330.

${ }^{4}$ Sur cette ambiguïté, cf., en dernier lieu, Dugan 2005, p. 172-250, en part. p. 248-250 et d'une façon générale la question du sens politique du dialogue, $c f$. infra p. 38 n.90.

${ }^{5} \mathrm{Cf}$. en part. de orat. I, 33 .
} 
quales fuissent ${ }^{6}$. C'étaient eux en effet qui de progrès en progrès lui avaient donné son importance. Toutefois, les personnages dont il était question étaient autant des hommes politiques que des orateurs. Ils n'étaient l'un que parce qu'ils étaient l'autre. Et leur place dans l'éloquence tenait à leur place dans la cité. Nous allons le vérifier en examinant la méthode que suivit Cicéron pour le faire apparaître.

Le schéma chronologique auquel Cicéron se conformait a été étudié et discuté depuis longtemps. On y voyait une organisation plus ou moins stricte jusqu'à ce que G.V. Sumner ${ }^{7}$ traite vraiment le sujet et révèle avec précision le plan que Cicéron avait adopté. Il montrait alors que la succession des orateurs s'organisait autour d'un certain nombre de personnages importants qu'il définissait comme des «key figures ». Les autres s'aggloméraient autour d'eux pour constituer des générations ou aetates. Ainsi, pour ne prendre que ces deux exemples, Cicéron employait-il des formules de transition de ce genre : Coniunctus igitur Sulpici aetati $P$. Antistius fuit $[\ldots]^{8}$; ou un peu plus loin : L. Sisenna $[\ldots]$ interiectusque inter duas aetates Hortensi et Sulpici [...] ${ }^{9}$ qui permettaient de définir la situation des orateurs par rapport aux personnalités dominantes. C'était en y faisant référence qu'il plaçait en effet les personnages de moindre relief comme par exemple tous ceux qui étaient à peu près [20] contemporains de Caton l'ancien : cum hoc Catone grandiores natu $[\ldots]^{10}$ de minoribus autem $[\ldots]^{11}$ sed uiuo Catone minores natu $[\ldots]^{12}$.

Il rassemblait enfin en conclusion tous ces personnages dans une sorte de bilan rétrospectif qui rappelait les grands moments de l'éloquence romaine. Mais cela lui permettait aussi d'affirmer que chaque génération avait été dominée par un ou deux personnages qui l'avaient emporté sur les autres :

\begin{abstract}
Nonne cernimus uix singulis aetatibus binos oratores laudabilis constitisse? Galba fuit inter tot aequalis unus excellens, cui, quem ad modum accepimus, et Cato cedebat senior et qui temporibus illis aetate inferiores fuerunt ; Lepidus postea, deinde Carbo ; nam Gracchi in contionibus multo faciliore et liberiore genere dicendi, quorum tamen ipsorum ad aetatem laus eloquentiae perfecta non fuit; Antonius, Crassus, post Cotta, Sulpicius, Hortensius $[\ldots]^{13}$.

Ne voyons-nous pas, d'ailleurs, que c'est à peine si à chaque génération il surgit deux orateurs de valeur? Galba fut seul à exceller parmi le grand nombre de ses contemporains et la tradition nous apprend qu'il ne fut égalé ni par Caton, qui était plus vieux que lui, ni par deux autres contemporains, qui étaient plus jeunes, Lepidus et ensuite Carbo. Je ne dis rien des Gracques, dont les harangues ont sans doute quelque chose de plus facile et de plus dégagé, mais qui disparurent avant que leur talent oratoire eût donné toute sa mesure. Puis vinrent Antoine et Crassus, après eux Cotta, Sulpicius, Hortensius [...].
\end{abstract}

\footnotetext{
${ }^{6}$ Brut. 20. Sur le Brutus comme histoire littéraire et les évolutions qui l'ont marquée, cf. Vogt-Spira 2000.

${ }^{7}$ Sumner 1973, p. 3-10 ; p. 151-154 qui donne l'état des discussions antérieures. Cf. aussi les remarques de Narducci 1997, p. 113.

${ }^{8}$ Brut. 226.

${ }^{9}$ Brut. 228.

${ }^{10}$ Brut. 77.

${ }^{11}$ Brut. 78.

${ }^{12}$ Brut. 80.

${ }^{13}$ Brut. 333 ; trad. J. Martha.
} 
Pour Cicéron en effet, l'éloquence n'était pas seulement un mode d'expression et d'action civique, c'était aussi un espace de compétition.

Avant de reprendre ce dernier point, reprenons la liste des figures dominantes et tentons de cerner leur personnalité. Telle que l'a établie G.V. Sumner, les aetates se seraient ainsi succédé : aetas de Caton l'ancien (consul en 195, né en 234), aetas de Ser. Sulpicius Galba (cons. en 144, né vers 191), aetas de M. Aemilius Lepidus Porcina (cons. en 137, né vers 180), aetas de C. Papirius Carbo (cons. en 120, né vers 163) et des Gracques, Tiberius (tr. pl. en 133, né en 163) et Caius (tr. pl. en 123, né en 154-3), aetas de Q. Lutatius Catulus (cons. en 102, né en 149), aetas de M. Antonius (cons. en 99, né en 143) et de L. Licinius [21] Crassus (cons. en 95, né en 140), aetas de C. Iulius Caesar Strabo Vopiscus (cand. cons. pour 88, né en 131 ou en 127) aetas de P. Sulpicius Rufus (tr. pl. en 88, né en 124-3) et de C. Aurelius Cotta (cons. en 75, né en 124) et enfin aetas de Q. Hortensius Hortalus (cons. en 69, né en 114).

Cicéron s'arrêtait là, mais si l'on suit G.V. Sumner dans sa reconstitution, deux autres générations se dessinaient de façon implicite : celle de Cicéron lui-même (cons. en 63, né en 106) et celle de M. Iunius Brutus (prét. en 42, né en 78). Et je me demande s'il ne faudrait pas distinguer aussi une aetas de César (cons. en 59, né en 100) et de M. Claudius Marcellus (cons. en 51, né vers 95) avant celle de Brutus, et une aetas de M. Cornelius Cethegus (cons. en 204, né vers 241) au début du dialogue.

Cicéron se permettait certes quelques digressions sur des catégories particulières qui avaient marqué leur époque : les orateurs populares qui avaient succédé aux Gracques ${ }^{14}$ et, de façon plus nette encore, les orateurs d'origine municipale qui s'étaient fait connaître dans les années qui précédaient la guerre sociale ${ }^{15}$. Mais à ces quelques variations près, le dialogue se déroulait ainsi d'aetas en aetas. Il faut bien prendre garde cependant qu'il ne s'agissait pas de générations qui auraient régulièrement scandé le déroulement du temps. Même si les écarts entre deux d'entre elles étaient généralement d'une dizaine d'années, ils pouvaient parfois fortement varier : 43 ans séparaient la naissance de Caton de celle de Sulpicius Galba, alors que celles de Lutatius Catulus et de Caius Gracchus n'étaient éloignées que de 5 ans. Les groupes étaient ainsi moins définis en classes d'âge qu'ils ne l'étaient par la place qu'ils occupaient dans l'histoire de l'éloquence par les changements qu'ils avaient apportés ou connus.

En effet, la caractéristique essentielle des personnages qui constituaient les «key figures » tenait certes à ce qu'ils l'emportaient sur leurs contemporains par la qualité de leur art oratoire, mais c'était aussi souvent parce qu'ils avaient apporté quelque chose de plus que leurs prédécesseurs et qu'ils étaient devenus des modèles par leur capacité d'innovation. Sans entrer dans le détail, on peut rapidement rappeler ce que chacun d'eux avait pu, selon Cicéron, apporter à l'éloquence.

M. Cornelius Cethegus aurait été le premier à avoir été véritablement eloquens ${ }^{16}$. Caton associait l'élégance à l'efficacité et constituait certainement le premier modèle digne d'être imité $^{17}$. Ser. Sulpicius Galba avait apporté de [22] la uis et de la grauitas. Il avait eu, pour la première fois, recours à la miseratio et avait véritablement introduit l'émotion dans

\footnotetext{
${ }^{14}$ Brut. 224-225.

${ }^{15}$ Brut. 169-172.

${ }^{16}$ Brut. 58 : Ennius [...] et oratorem appellat et suauiloquentiam tribuit.

${ }^{17}$ Brut. 63 : Catonis autem orationes [...] acuti sunt, elegantes, faceti breues. Brut. 65 : quis illo grauior in laudando, acerbior in uituperando, in sententiis argutior, in docendo edisserendoque subtilior? [...] omnes oratoriae uirtutes in eis [sc. orationibus] reperientur.
} 
l'éloquence ${ }^{18}$. M. Aemilius Lepidus Porcina mit du soin dans la construction de la phrase ${ }^{19}$. Il fut aussi le premier dont on peut identifier les élèves, C. Papirius Carbo et Tiberius Gracchus $^{20}$. Peut-être est-ce donc avec lui qu'apparut le tirocinium fondé sur la transmission d'une compétence recherchée et non pas sur la parenté. Avec les Gracques et Papirius Carbo qui bénéficièrent d'un haut degré de formation ${ }^{21}$ l'éloquence franchit une première étape, grâce à la capacité d'user de tous les niveaux de style.

La génération suivante de M. Antonius et L. Licinius Crassus fut celle que Cicéron idéalisa dans le De oratore. Au milieu de multiples qualités, M. Antonius semble s'être distingué par l'importance accordée au choix des figures, à la memoria et l'actio ${ }^{22}$ et Crassus semble l'avoir fait par l'association de la grauitas et de l'elegantia, et par l'abondance (copia) des discours ${ }^{23}$ qui, selon Cicéron, était la qualité qui donnait le plus de prix à l'art oratoire ${ }^{24}$. Avec ces maximi oratores, l'éloquence romaine parvenait à un premier niveau de maturité et atteignait enfin le niveau de la Grèce ${ }^{25}$. C. Iulius Caesar Strabo qui vint [23] après eux l'emportait par son sens de l'humour et son urbanitas $^{26}$. C. Aurelius Cotta et P. Sulpicius Rufus se distinguaient, le premier par son inuentio et son elocutio $^{27}$, le second par son action particulièrement efficace ${ }^{28}$. Hortensius enfin l'emportait dans son art de l'inuentio et de la dispositio, mais il y ajoutait aussi les qualités nécessaires aux autres parties de l'art oratoire, l'actio, la memoria et atteignait véritablement la copia par son talent ${ }^{29}$.

Ainsi se succédaient les orateurs qui avaient fait l'éloquence romaine. Cicéron suivait le schéma qu'Atticus avait adopté dans cet ouvrage qu'il venait de publier et dont son ami s'inspirait ${ }^{30}$. Il avait pour effet de faire acteurs de l'Histoire les hommes politiques eux-mêmes qui avaient apporté leur contribution à la grandeur de Rome.

\footnotetext{
${ }^{18}$ Brut. 82 : ut egrederetur a proposito ornandi causa, ut delectaret animos aut permoueret, ut augeret rem, ut miserationibus, ut communibus locis uteretur (cf. tous les paragraphes 82-94).

${ }^{19}$ Brut. 96: Hoc in oratore Latino primum mihi uidetur et leuitas apparuisse illa Graecorum et uerborum comprensio et iam artifex, ut ita dicam, stilus.

${ }^{20}$ Brut. 96.

${ }^{21}$ Brut. 104 (Ti. Gracchus) : [...] a puero doctus et Graecis litteris eruditus [...].Brut. 105 (Carbo) : [...] canorum oratorem et uolubilem et satis acrem atque eundem et uehementem et ualde dulcem et perfacetum fuisse [sc. L. Gellius] dicebat ; [...] et in exercitationibus commentationibusque multum operae solitum esse ponere (il est donc le premier dont on sache qu'il ait fait usage des déclamations) ; Brut. 125 (C. Gracchus) : [...] doctus a puero [...] noli enim putare quemquam [...] pleniorem aut uberiorem ad dicendum fuisse. Brut. $126:[. .$.$] grandis$ est uerbis, sapiens sententiis, genere toto grauis.

${ }^{22}$ Brut. 139 : erat memoria summa [...] ; Brut. 140 : uerum multo magis hoc idem in sententiarum ornamentis et conformationibus [...] ; Brut. 141 : sed cum haec magna in Antonio tum actio singularis [...].

${ }^{23}$ Brut. 143 : Erat summa grauitas, erat cum grauitate iunctus facetiarum et urbanitatis oratorius [...] argumentorum et similitudinum copia. [...] sic in interpretando in definiendo in explicanda aequitate nihil erat Crasso copiosius [...].

${ }^{24}$ Cf. en part. de orat. I, 50 ; 59 ; II, 120 ; III, 104 ; orat. 97.

${ }^{25}$ Brut. 138.

${ }^{26}$ Brut. 177 : Festiuitate [...] et facetiis [...] et superioribus et aequalibus suis omnibus praestitit [...], nemo unquam urbanitate, nemo lepore, nemo suauitate conditior.

${ }^{27}$ Brut. 202 : inueniebat igitur acute Cotta, dicebat pure ac solute.

${ }^{28}$ Brut. 203 : fuit [...] omnium uel maxume, quos quidem ego audiuerim, grandis et [...] tragicus orator.

${ }^{29}$ Brut. 302 : duas quidem res quas nemo alius, partitiones, quibus de rebus dicturus esset, et conlectiones memor et quae essent dicta [...].Brut. 303 : Erat in uerborum splendore elegans, compositione aptus, facultate copiosus [...]. Rem complectebatur memoriter, diuidebat acute [...]. Cf. sur ce point infra, p. 146 sq., les analyses d'A. Garcea et V. Lomanto.

${ }^{30}$ Brut. 13-16 ; 19 ; 42 ; cf. orat. 120 ; Beck, Walter 2004, p. 358-367.
} 
L'objectif de Cicéron était de décrire cette ascensio dans l'art oratoire et de montrer par quels efforts elle pouvait conduire à la perfectio et à l'absolutio :

\begin{abstract}
Est enim propositum colligere eos, qui hoc munere in ciuitate functi sint, ut tenerent oratorum locum ; quorum quidem quae fuerit ascensio et quam in omnibus rebus difficilis optimi perfectio atque absolutio ex eo quod dicam existimari potest $t^{31}$.

C'est que mon dessein est de rassembler tous ceux qui par leur rôle dans la cité ont été conduits à faire acte d'orateurs. Ce qu'a été leur montée graduelle <vers l'éloquence>, et combien en toutes choses il est difficile d'atteindre et de réaliser ce qu'il y a de mieux, la simple remarque que voici le fera comprendre.
\end{abstract}

Bien entendu, cette ascension avait son sommet. C'était Cicéron qui, faux modeste, suggérait qu'il s'agissait de lui-même. Ainsi - expliquait-il - le premier degré de maturité que l'éloquence avait atteint avec Antonius et Crassus [24] en annonçait un autre, où l'art oratoire s'enrichirait encore davantage de philosophie, de droit et d'histoire, et que, comme il le faisait dire à Brutus ${ }^{32}$, Cicéron lui-même incarnait ${ }^{33}$. Surtout, c'était à César qu'il attribuait l'hommage le plus décisif quand il reprenait et développait cet élogeoù le vainqueur des Gaules reconnaissait que celui qui avait introduit l'abondance oratoireavait fait honneur au nom et à la dignité du peuple romain ${ }^{34}$ :

\begin{abstract}
Plus enim certe adtulit huic populo dignitatis, qui non illustrauit modo sed etiam genuit in hac urbe dicendi copiam, quam illi qui Ligurum castella expugnauerunt : ex quibus multi sunt, ut scitis, triumphi ${ }^{35}$.

Assurément il a fait plus d'honneur à notre peuple l'homme, quel qu'il soit, s'il est vrai qu'il existe, qui non seulement a mis en lumière, mais encore a créé à Rome l'abondance oratoire, que ceux qui ont enlevé des bicoques liguriennes, bicoques dont la prise a été, comme vous le savez, le prétexte de maints triomphes.
\end{abstract}

L'énumération trouvait là une conclusion magnifique. Non seulement Cicéron parachevait de sa personnalité la progression de l'art oratoire romain vers la perfection, mais il prétendait même qu'il l'emportait en gloire et en intérêt pour la cité sur les autres vertus qui définissaient la supériorité aristocratique.

Cette première fonction des figures dominantes où chacune d'entre elles recevait la responsabilité d'une contribution aux progrès de l'éloquence, n'explique pas tout. D'abord, parce que cette première conclusion mérite d'être nuancée. Certains orateurs furent de

\footnotetext{
${ }^{31}$ Brut. 137 ; trad. J. Martha.

${ }^{32}$ Brut. 161 : ut eo nihil ferme quisquam addere posset, nisi qui a philosophia a iure ciuili ab historia fuisset instructior. Brut. 162 : Erit, inquit [M.] Brutus aut iam est iste quem exspectas?

${ }^{33}$ Cf. surtout Brut. 322.

${ }^{34}$ Brut. 253.

${ }^{35}$ Brut. 255 ; trad. J. Martha. Cf. également Brut. 254 : Tum Brutus : amice hercule, inquit, et magnifice te laudatum puto, quem non solum principem atque inuentorem copiae dixerit, quae erat magna laus, sed etiam bene meritum de populi Romani nomine et dignitate. Quo enim uno uincebamur a uicta Graecia, id aut ereptum illis est aut certe nobis cum illis communicatum. Brut. 255: Hanc autem, inquit, gloriam testimoniumque Caesaris tuae quidem supplicationi non, sed triumphis multorum antepono.
} 
véritables initiateurs. Sulpicius Galba par exemple osa la miseratio, et cela fit scandale ${ }^{36}$. Mais on voit mal ce que d'autres, excellents par ailleurs, apportèrent de nouveau. Cicéron [25] luimême le signifiait, en laissant entendre que l'éloquence qui était parvenue à un premier niveau de maturité avec Antonius et Crassus, n'atteindrait le sommet qu'avec lui-même ${ }^{37}$. Les autres qui se situaient dans l'intervalle, Aurelius Cotta, Sulpicius Rufus et Hortensius, étaient donc de très grands orateurs mais n'étaient pas en position de faire faire des progrès décisifs, ou pour le moins véritablement identifiables, à l'art oratoire. La seconde raison tenait à ce que, pour Cicéron, l'histoire de l'éloquence reposait aussi sur les autres, les orateurs secondaires. Son objectif était, comme on l'a vu, de rassembler qui hoc munere in ciuitate functi sint, ut tenerent oratorum locum ${ }^{38}$. Il s'attachait donc à les évoquer en grand nombre même si ses interlocuteurs le lui reprochaient parfois en considérant que ceux qu'ils citaient n'étaient pas dignes d'être retenus ${ }^{39}$.

Cette démarche narrative - retenir tous les orateurs et distinguer parmi eux les plus grands - avait un sens.

Elle s'inscrivait d'abord dans une conception romaine de l'Histoire qui plaçait au cœur du schéma narratif les grands hommes qui avaient gouverné la cité ${ }^{40}$. Ils constituaient autant de modèles dont l'exemplarité s'imposait dans la définition des comportements vertueux et fondait la légitimité aristocratique. La concaténation de leurs actes et de leurs figures avait pour double effet de rendre compte, dans la diachronie, de la construction de la puissance de Rome et d'établir, par la répétition de leurs exploits, le code des conduites qui définissaient le magistrat et sénateur romain soucieux de la grandeur de sa famille et de sa cité. Bien entendu, la compétition pour la gloire qui animait tous ces protagonistes de la vie politique déterminait aussi bien les motivations réelles des acteurs que les reconstitutions des historiens et la nécessité de devoir l'emporter aussi bien sur ses propres ancêtres que sur ses contemporains et concurrents créait les conditions de l'émulation dans la vertu qui était, pensait-on, à l'origine de la supériorité romaine.

Les racines de ce trait essentiel de la culture politique aristocratique étaient à la fois profondes et anciennes. Elles étaient antérieures à l'émergence même de l'écriture romaine de l'Histoire et trouvaient leur première manifestation identifiable dans les célébrations par les grandes familles des exploits de leurs membres. La plus évidente était bien entendu cette pratique des funérailles qui avait été décrite par Polybe et qui consistait en cette procession des [26] figurants représentant les ancêtres du défunt porteurs des insignes de leur pouvoir dans la succession des magistratures qu'ils avaient accomplies. Elle était soutenue par la laudatio funebris prononcée par son descendant le plus proche qui tout à la fois inscrivait son action au service de la cité dans la mémoire civique et contribuait à la définition collective des comportements exemplaires $^{41}$. Ce mode de célébration des grands hommes dont la vertu avait fait Rome ne manqua pas de prendre sa place dans les pratiques littéraires dès lors que cellesci trouvèrent une expression autonome ${ }^{42}$. Les annales d'Ennius constituèrent sans doute une

\footnotetext{
${ }^{36}$ Cf. en part. Brut. 89-90 ; de orat. I, 227-228 ; Liv., perioch. 49 ; Val. Max. VIII, 1 abs. 2 ; Quint., inst. II, 15, 8 .

${ }^{37}$ Brut. 161-162, cité supra, p. 25, n. 32.

${ }^{38}$ Brut. 137.

${ }^{39} \mathrm{Cf}$. par ex. Brut. 176 : sed ab eis qui tantum in dicentium numero, non in oratorum fuerunt, iam ad oratores reuortamur. Censeo, inquit, Atticus ; eloquentis enim uidebare, non sedulos uelle conquirere ; 181-182 ; 244 ; $251 ; 269-270 ; 299$.

${ }^{40} \mathrm{Cf}$. surtout les analyses de Walter 2004.

${ }^{41}$ C'est tout le mode de célébration des ancêtres qu'il faudrait évoquer, cf. Flower 1996, p. 91-158.

${ }^{42}$ Sur la place des vertus dans l'épopée et l'histoire, cf. en part. Mutschler 2000.
} 
étape décisive, car elles l'inscrivaient dans la succession diachronique des événements dont ils étaient les auteurs ${ }^{43}$. Les annalistes reprirent un schéma analogue même si sans doute l'intérêt pour le déroulement des différents épisodes prenait davantage de place. Un processus se mettait ainsi en place qui faisait que ces vertus dont les membres de la noblesse revendiquaient la possession définissaient en fin de compte le mos maiorum dans un horizon commun des normes de comportement ${ }^{44}$.

Cicéron s'inscrivait bien entendu dans ce schéma général, d'abord parce que le contexte de production littéraire de la deuxième moitié du ${ }^{\mathrm{er}}$ siècle faisait apparaître un intérêt particulier pour les reconstitutions de listes de magistrats ou de grands hommes. Lui-même, on l'a vu, s'était appuyé sur le liber annalis de son ami Atticus ${ }^{45}$. À peu près au même moment et dans les années qui suivirent, d'autres ouvrages, au demeurant mal connus, apparurent qui mettaient en série des biographies ou des épisodes exemplaires et qui déterminaient par leur rassemblement le catalogue des vertus. On relèvera la publication du liber annalis de Scribonius Libo, celle des biographies de Cornelius Nepos, celle des Hebdomades ou Imagines de Varron, celle du de uiris illlustribus de Santra ou celle encore des recueils d'exempla d'Hygin ${ }^{46}$. On rappellera surtout que, dans son Forum, Auguste avait reproduit ce même schéma des uiri illustres se succédant les uns aux autres dans la construction [27] de la puissance de Rome en faisant converger la série des statues des membres de sa famille avec celle des grands hommes de l'histoire romaine, dont la rencontre légitimait son propre pouvoir ${ }^{47}$.

La démarche qu'il adoptait dans la construction du Brutus s'inscrivait donc dans un contexte bien établi de représentation de l'Histoire de la cité. Lui-même, autant que l'état fragmentaire du dialogue permet de le savoir, l'avait déjà adopté dans le De republica dont l'un des objectifs revenait à définir l'homme d'État idéal. C'était la succession de ceux qui, chacun à son tour, avaient apporté à la constitution romaine une contribution solide et clairvoyante, sage pour tout dire, qui en avaient fait l'équilibre et la soliditét ${ }^{48}$. L'histoire de l'éloquence reproduisait le même schéma. D'abord parce qu'il permettait de structurer l'image d'un progrès engendré par les ambitions et les efforts successifs des différents orateurs. Ensuite parce qu'il faisait de l'art oratoire une forme particulière de l'action civique dont la maîtrise croissante et la floraison finale accompagnaient la construction de la puissance de Rome.

Tous les orateurs avaient donc leur place dans cette narration, dans la mesure où les rassembler tous permettait de distinguer les plus grands. Une telle réunion reposait aussi sur l'idée que l'éloquence était un art difficile. Ceux qui s'y consacraient s'investissaient grâce à lui dans la vie de la cité et leur effort méritait d'être signalé, même quand il n'avait pas permis d'atteindre les plus grandes réussites :

\footnotetext{
${ }^{43}$ Cf. Gildenhard 2003; Walter 2004, p. 258-279 ; Cicéron s'appuyait lui-même directement sur Ennius (en part. Brut. 57-59; $71 ; 75-76)$.

${ }^{44}$ Sur ce processus, cf. en part. Hölkeskamp 1996 ; Blösel 2000 et Mencacci 2001.

${ }^{45}$ Cf. supra p. 24, n. 30.

${ }^{46}$ Sur ces auteurs, cf. Schanz Hosius 1927,p. 323-324 ; 351-361 ; 561-563 ; 584 ; Schanz Hosius 1935, p. 368372 ; Rawson 1985, p. 220-221; 230-232; 198-199. Sur le recours de Cicéron à la littérature antiquaire, cf. Rawson 1972 ; sa méthode et ses intérêts cf. Walter 2004, p. 357-373.

${ }^{47}$ Sur ce point cf. surtout, Zanker 1987, p. 213-217.

${ }^{48}$ Cf . rep. I, 1-2 ; II, 2-63 ; en part. 22 ; 30 ; 33 ; $51: 55$; III, 4-7 et aussi Brut. 19.
} 
De his autem, quos ipsi uidimus, neminem fere praetermittimus eorum quos aliquando dicentes audiuimus. Volo enim sciri in tanta et tam uetere re publica maximis praemiis eloquentiae propositis omnes cupisse dicere, non plurimos ausos esse, potuisse paucos ${ }^{49}$.

Par contre, parmi les hommes de mon temps, je ne laisse de côté presque aucun de ceux que j'ai entendus parler. Car je veux que l'on sache que dans une si grande et si ancienne république, où les plus brillantes récompenses ont toujours été proposées à l'éloquence, tous ont eu l'ambition de parler en public, assez peu ont osé le faire et peu en ont été capables.

Cette première étape dans la démarche avait pour effet, tout en les évoquant en parallèle, de distinguer les orateurs des autres hommes politiques qui avaient [28] construit leur position sociale sur d'autres types d'action civique, la jurisprudence et l'art militaire notamment.

Elle permettait ensuite, en relevant les meilleurs parmi toute cette foule, de faire apparaitre ceux qui y avaient plus réussi que les autres et l'emportaient en quelque sorte sur leurs contemporains. Le grand orateur ne marquait pas seulement une étape dans la chaîne du temps. Il dominait aussi son époque comme un exemple particulier de succès et s'imposait comme modèle. Ainsi la notion de génération ne devait-elle pas simplement être prise dans son sens diachronique. Elle correspondait véritablement au groupe des orateurs d'une époque donnée qu'une ou deux personnalités plus fortes que les autres structuraient autout d'eux.

Les exemples de ce mode de construction abondent. Ainsi à propos de Sulpicius Galba : sed inter hos aetate paulum his antecedens [...] eloquentia praestitit ${ }^{50}$; illius aetatis principem $^{51}$. De Papirius Carbo : Hic optimus illis temporibus est patronus habitus $[. . .]^{52}$. De C. Iulius Caesar Strabo : Festiuitate [...] et facetiis [...] et superioribus et aequalibus suis omnibus praestitit [...] $]^{53}$. D'Aurelius Cotta et de Sulpicius Rufus : Ex his [...] tum omnium facile primas tulerunt ${ }^{54}$; uterque aequalibus suis plurimum praestitit ${ }^{55}$; His duobus eiusdem aetatis adnumerabatur nemo tertius ${ }^{56}$. Ou encore, d'Hortensius : suos inter aequales [...] longe praestitit $^{57}$; facile primas tenebat adulescens ${ }^{58}$.

La prééminence que ces personnages s'étaient gagnée n'était évidemment pas absolue. Des hiérarchies se créaient. Derrière les premiers, on trouvait des seconds et des troisièmes. Ainsi après M. Antonius et L. Licinius Crassus, venait L. Marcius Philippus : L. Philippus proxumus accedebat, sed longo interuallo tamen proxumus ${ }^{59}$. Et de la même façon, C. Scribonius Curio se tenait derrière Aurelius Cotta et Sulpicius Rufus : Erant tamen quibus uideretur illius aetatis tertius Curio $[\ldots]^{60}$. C'était évidemment la comparaison qui permettait

\footnotetext{
${ }^{49}$ Brut. 181-182 ; trad. J. Martha.

${ }^{50}$ Brut.. 82.

${ }^{51}$ Brut.. 295.

${ }^{52}$ Brut.. 106.

${ }^{53}$ Brut.. 177.

${ }^{54}$ Brut.. 183.

${ }^{55}$ Brut.. 204.

${ }^{56}$ Brut.. 207.

${ }^{57}$ Brut.. 230.

${ }^{58}$ Brut.. 327.

${ }^{59}$ Brut. 173 ; cf. 186.

${ }^{60}$ Brut.. 210.
} 
d'obtenir un tel classement. Elle était constante. Voici par exemple comment Cicéron confrontait les qualités de L. Licinius Crassus et de Q. Mucius Scaevola : [29]

\begin{abstract}
Nam ut paulo ante dixi consultorum alterum disertissimum, disertorum alterum consultissimum fuisse si in reliquis rebus ita dissimiles erant inter se, statuere ut tamen non posses utrius te malles similiorem ${ }^{61}$.

Ces personnages, qui étaient, comme je l'ai dit tout à l'heure, l'un le plus éloquent des jurisconsultes, l'autre le meilleur jurisconsulte parmi les hommes éloquents, étaient, sous tous les autres rapports, très différents l'un de l'autre, et cependant <si parfaits l'un et l'autre> qu'on n'eût pas su dire auquel des deux on eût mieux aimé ressembler.
\end{abstract}

Et surtout elle était complètement voulue et assumée par les protagonistes eux-mêmes qui s'y prêtaient complètement. On peut relever cette indication intéressante à propos de Laelius et de Scipion l'Africain :

Sed est mos hominum ut nolint eundem pluribus rebus excellere. Nam ut ex bellica laude aspirare ad Africanum nemo potest [...], sic ingeni, litterarum, eloquentiae, sapientiae denique etsi utrique primas, priores tamen libenter deferunt Laelio. Nec mihi ceterorum iudicio solum uidetur, sed etiam ipsorum inter ipsos concessu ita tributum fuisse ${ }^{62}$.

Mais les hommes sont ainsi faits qu'ils n'aiment pas voir une même personne exceller dans plusieurs choses à la fois. Si pour le talent militaire personne ne peut songer à une comparaison avec l'Africain [...], pour la vivacité de l'esprit, la culture littéraire, l'éloquence, la philosophie, tout en regardant ces deux hommes comme les premiers des Romains, on regarde volontiers Laelius comme le premier des deux. Et ce n'est pas seulement l'opinion publique qui a fait ce partage de gloire : eux-mêmes paraissent l'avoir fait entre eux.

Mais elle apparaissait surtout dans les relations entre Cicéron et Hortensius. Ainsi, après 63,

[...] cum iam paene euanuisset Hortensius et ego anno meo [...] consul factus essem, reuocare se ad industriam coepit, ne cum pares honore essemus, aliqua re superiores uideremur $^{63} \cdot[30]$

[...] Hortensius avait presque disparu lorsqu'arrivé à l'âge légal [...], je fus fait consul à mon tour. À ce moment, il se remit au travail, de peur qu'étant devenu son égal en dignité, je ne parusse avoir en quelque chose une supériorité sur lui.

Les grands orateurs qui servaient de modèles ne l'étaient donc que parce qu'ils l'avaient emporté sur les autres dans un processus de comparaison et de distinction pour la supériorité oratoire. En ce sens, la compétition pour être le meilleur ne se séparait pas des autres modes de rivalité aristocratique. Il fallait l'emporter sur ses contemporains et ses prédécesseurs, rayonner sur sa génération et servir soi-même de modèle aux autres plus jeunes.

\footnotetext{
${ }^{61}$ Brut.. 148 ; trad. J. Martha. Cf. Brut.. 145 et parmi les références à la causa Curiana, Brut.. 197-198; de orat. I, 180 ; 242-243 ; II, 220-221.

${ }^{62}$ Brut. 84 ; trad. J. Martha.

${ }^{63}$ Brut.. 323 ; trad. J. Martha.
} 
Qui cependant devait en juger? Quelle instance d'évaluation avait compétence et légitimité pour décider qui était le meilleur de sa génération ? Cicéron lui-même pour commencer. C'était lui en effet qui choisissait de retenir tel ou tel orateur dans son énumération, rappelait ses mérites, soulignait ses défauts et lui attribuait son rang. Il s'appuyait parfois sur son expérience personnelle, particulièrement dans le cas des orateurs qui dominaient le forum au temps de sa jeunesse ${ }^{64}$. Cela ne suffisait évidemment pas, puisqu'il était loin d'avoir connu tous les personnages qu'il citait. Il renvoyait donc souvent à une opinion commune, le plus souvent anonyme dont le jugement s'exprimait au travers de termes passifs dépourvus de sujets identifiables : probatus, habitus, numeratus ${ }^{65}$, mais qui, en quelque sorte, définissaient le jugement des contemporains.

La notion peut sembler un peu vague. Elle ne l'était pas. D'abord parce qu'elle était diversifiée. Cicéron distinguait en effet entre le jugement du public et celui des spécialistes qui parfois s'écartaient l'un de l'autre. Ainsi, à propos de son cousin C. Visellius Varro : in quo fateor uolgi iudicium a iudicio meo disensisse ${ }^{66}$, ou à propos du déclin d'Hortensius après son consulat: quantum non quiuis unus ex populo, sed existumator doctus et intellegens posset cognoscere ${ }^{67}$ ou encore à propos du même Hortensius, ces appréciations divergentes des membres de l'aristocratie, jeunes et vieux, et du peuple: Non probabantur haec senibus [...] sed mirabantur adulescentes, multitudo mouebatur ${ }^{68}$. Les critères de jugement n'étaient donc pas les mêmes selon qu'il s'agissait d'auditeurs [31] savants bien formés aux règles de la rhétorique qui appréciaient en connaisseurs la construction et la mise en scène des discours et les simples citoyens qui en subissaient les effets sans trop chercher à comprendre ce qui les produisait.

On pourrait supposer qu'entre l'écoute critique et l'écoute naïve, ce serait la première qui, pour Cicéron, fournirait le jugement le plus pertinent. Bien au contraire et ce point est important : c'était l'avis populaire qui décidait de tout et en particulier de la position d'un orateur.

Il consacrait en effet à cette question un excursus ${ }^{69}$ un peu fourni que l'on ne peut pas complètement reproduire ici, mais dont les conclusions étaient claires. Pour Cicéron, c'était l'effet produit par un orateur qui pouvait décider de sa qualité ${ }^{70}$. Rien d'autre, et de cela même les spécialistes devaient convenir ${ }^{71}$, car les discours étaient destinés au peuple et c'était son adhésion qui témoignait ou non de leur qualité ${ }^{72}$. Lui aussi procédait par comparaison, puisque dans les contextes judiciaires ou politiques qui les amenaient à s'opposer, les orateurs se disputaient son assentiment. Cicéron prenait l'exemple de la causa Curiana où Q. Mucius Scaevola et L. Licinius Crassus s'étaient opposés et montrait qu'il n'était pas douteux que le

\footnotetext{
${ }^{64}$ Cf. Brut. 304-305 et $181 ; 203 ; 207 ; 213 ; 259 ; 265$.

${ }^{65}$ Cf. par ex., Brut.. 78 (C. Sulpicius Galus, cons. en 166) : oratorum in numero est habitus ; Brut.. 98 (P. Licinius Crassus, cons. en 131) : ualde probatum oratorem ; Brut.. 221 (C. Papirius Carbo, prét. vers 83) : non satis acutus orator, sed tamen orator numeratus est.

${ }^{66}$ Brut.. 264.

${ }^{67}$ Brut.. 320.

${ }^{68}$ Brut.. 326.

${ }^{69}$ Brut.. 183-200.

${ }^{70}$ Brut.. 184 : qualis uero sit orator ex eo, quod is dicendo efficiet, poterit intellegi.

${ }^{71}$ Brut.. 188 : quod enim probat multitudo, hoc idem doctis probandum est. Cf. magré tout le cas de Fimbria, Brut.. 233.

${ }^{72}$ Brut. 191 : poema enim reconditum paucorum adprobationem, oratio popularis adsensum uolgi debet mouere.
} 
second qui l'avait emporté, était nécessairement le meilleur aussi bien aux yeux des savants qu'aux yeux du peuple ${ }^{73}$.

Cette position centrale de l'auditoire dans l'évaluation de l'éloquence, cette primauté réservée à l'efficacité permettaient à Cicéron de justifier les choix qu'il opérait. C'était en fonction de la trace que l'Histoire avait conservée des plus anciens, de l'effet que leurs discours avaient pu produire, qu'il pouvait distinguer les orateurs qui méritaient de l'être dans les générations les plus anciennes, celles dont lui-même ou d'autres plus anciens encore ne pouvaient avoir conservé la mémoire ${ }^{74}$. C'était aussi en fonction des choix populaires qu'il donnait la préférence aux meilleurs de chaque génération et qu'il parvenait à assigner à chacun d'eux sa position. Or, comme Cicéron le soulignait en prenant l'exemple de la causa Curiana, c'était dans le contexte de [32] compétition que créaient les affrontements politiques et judiciaires que le peuple pouvait trouver à exprimer ses préférences. L'évaluation à laquelle il procédait n'était donc pas pur jugement fondé sur le plaisir et l'intérêt. Elle passait par un véritable choix, qui devait s'opérer dans un contexte civique précis et qui revenait à déterminer qui avait tort ou raison. Le peuple romain, par ses préférences, exprimait sa confiance et jouait ce même rôle d'arbitre que dans les autres instances, électorales notamment, lui réservait la pratique institutionnelle romaine. Les grands orateurs s'inscrivaient en effet dans la série des grands hommes et le schéma narratif du Brutus reprenait naturellement celui de la concaténation des figures exemplaires.

C'était surtout le contexte judiciaire qui permettait cette détermination. Les membres de l'aristocratie avaient vocation à défendre leurs concitoyens en justice, c'est à dire à exercer la fonction de patronus. À la fin de la République, le caractère héréditaire du lien de patronat s'était affaibli et le choix était devenu relativement libre. Aussi, les Romains qu'un procès menaçait cherchaient-ils à se gagner l'assistance des orateurs les plus efficaces.

On trouve une première indication dans le fait que Cicéron employait parfois le terme de patronus comme un quasi-synonyme d'orator ${ }^{75}$. Il désignait par ce mot les personnages qui s'étaient révélés assez actifs et compétents pour défendre avec succès ceux qui avaient fait appel à eux (in patronis, in patronorum numero) ${ }^{76}$. Ils étaient assez nombreux pour que Cicéron finisse par employer le mot de turba ou celui de $u u l g u s^{77}$. Mais le groupe qu'ils constituaient avait aussi sa hiérarchie puisqu'il faisait allusion aux principes patronorum ${ }^{78}$, ceux dont précisément l'assistance était la plus recherchée.

C'était ainsi en effet que se définissaient les meilleurs, non pas par la qualité intrinsèque de leurs discours mais par leur efficacité. Cicéron ne perdait pas de vue que l'éloquence n'avait pas pour fonction d'obtenir les louanges d'un groupe de lettrés rompus aux règles de la rhétorique, mais de défendre des concitoyens en péril en forçant les sentences des juges. Ainsi étaient-ce en particulier les sollicitations des plaideurs qui définissaient le succès :

Cum multi essent oratores in uario genere dicendi, quis umquam ex his excellere iudicatus est uolgi iudicio, qui non idem a doctis probaretur? Quando autem dubium fuisset apud

\footnotetext{
${ }^{73}$ Brut.. 198 : Ab utroque autem causa perorata, si quaereretur uter praestaret orator, numquam profecto sapientis iudicium a iudicio uolgi discreparet.

${ }^{74}$ Cf. notamment Brut.. 53-56.

${ }^{75}$ Neuhauser 1958, en part. p. 187-202, mais qui a tort (p. 188-190) de voir dans le groupe des patroni du Brutus une catégorie professionnelle.

${ }^{76}$ Brut. $113 ; 124 ; 134 ; 136 ; 229 ; 233 ; 238 ; 243 ; 263 ; 319$.

${ }^{77}$ Brut.. $251 ; 332$.

${ }^{78}$ Brut.. 134 ; 233 ; 319 ; cf. 189 ; 207.
} 
patres nostros eligendi cui patroni [33] daretur optio, quin aut Antonium optaret aut Crassum? Aderant multi alii ; tamen utrum de his potius dubitasset aliquis, quin alterum nemo. Quid? adulescentibus nobis cum esset Cotta et Hortensius, num quis, quoi quidem eligendi potestas esset, quemquam his anteponebat ${ }^{79}$ ?

Parmi la foule des orateurs de toute espèce qui ont existé, en est-il un seul que l'opinion publique ait jugé excellent, sans que les savants aient confirmé cet arrêt ? Du temps de nos pères, quel est le citoyen qui, libre de choisir un défenseur, n'eût pas, sans hésiter un instant, désiré soit Antoine, soit Crassus? Il y avait beaucoup d'autres avocats et cependant, si l'on pouvait hésiter entre les deux, personnes n'eût hésité à choisir l'un des deux. Et dans notre jeunesse, quel homme, libre de son choix et pouvant prendre Cotta ou Hortensius, eût donné la préférence à quelqu'un d'autre ?

Elles opéraient aussi le classement, celui, précisément, qui déterminait la succession des modèles pour les générations des années 90-80:

Locus erat omnino in maxumis causis praeter eos de quibus supra dixi nemini ; propterea quod Antonius qui maxume expetebatur facilis in causis recipiendis erat; fastidiosor Crassus, sed tamen recipiebat. Horum qui neutrum habebat, confugiebat ad Philippum fere aut ad Caesarem ; Cotta <tum et $>$ Sulpicius expetebantur. Ita ab his sex patronis causae inlustres agebantur $[\ldots]^{80}$.

En tout cas, dans les grandes causes, à part les orateurs dont j'ai parlé plus haut, il n'y avait place pour personne: Antoine, le plus recherché de tous, acceptait volontiers les affaires qu'on lui apportait ; Crassus, un peu moins facile, ne les refusait pourtant pas. Quand on n'avait ni l'un ni l'autre pour avocat, on recourait presque toujours à Philippus ou à Caesar : après, c'est à Cotta et à Sulpicius qu'on allait. Ainsi, à eux six, ces avocats avaient toutes les causes importantes [...].

Le succès toutefois ne se mesurait pas seulement à l'activité judiciaire. Le public en effet ne séparait pas les divers champs de l'activité oratoire. [34]

La réussite dans les assemblées populaires attirait les requêtes d'assistance en justice. Ce fut la clé de l'ascension d'un personnage, au demeurant un peu secondaire, mais dont le destin fut en quelque sorte exemplaire :

Coniunctus igitur Sulpici aetati P. Antistius fuit rabula sane probabilis, qui multos cum tacuisset annos neque contemni solum, sed irrideri eiam solitus esset, in tribunatu primum, contra C. Iuli illam consulatus petitionem extraordinariam, ueram causam agens, est probatus; et eo magis, quod eandem causam cum ageret eius conlega ille ipse Sulpicius, hic plura et acutiora dicebat. Itaque post tribunatum primo multae ad eum causae, deinde omnes, maximae quaecumque erant deferebantur ${ }^{81}$.

Donc, à la génération de Sulpicius se rattache Publius Antistius, praticien très estimable. Il avait gardé le silence plusieurs années, s'étant vu l'objet, lorsqu'il parlait, des dédains et même des risées du public. Ce fut seulement lors de son tribunat que, combattant les

\footnotetext{
${ }^{79}$ Brut. 189 ; trad. J. Martha.

${ }^{80}$ Brut.. 207 ; trad. J. Martha.

${ }^{81}$ Brut. 226 ; trad. J. Martha.
} 
prétentions irrégulières de Caius Iulius $<$ Caesar $>$ au consulat et défendant la cause de la légalité, il remporta son premier succès, succès d'autant plus grand que dans cette cause, que soutenait avec lui son collègue le fameux orateur Sulpicius, c'était lui Antistius qui apportait les arguments les plus nombreux et les plus pénétrants. Aussi, à partir de son tribunat, fut-il chargé de beaucoup d'affaires ; puis un jour vint où toutes celles qui avaient de l'importance lui furent confiées.

Et réciproquement, la gloire obtenue devant les tribunaux qualifiait aux yeux du peuple et permettait de l'emporter aux élections. Ce fut, on le sait, tout le sens de la carrière de Cicéron et de bien d'autres encore, parmi les plus grands qui se distinguèrent par leur capacité oratoire et leur dévouement à leurs concitoyens. Le Brutus conserve çà et là la trace de ce lien en soulignant le succès inattendu que certains personnages tirèrent de leur activité de patronat. Ainsi, à propos d'un certain L. Turius, un homme nouveau dont l'énergie fut telle qu'il parvint malgré tout au seuil du consulat: paruo ingenio sed multo labore, quoquo modo poterat, saepe dicebat; itaque ei paucae centuriae ad consulatum defuerunt (en 64) ${ }^{82}$. Ou encore, cette carrière tronquée de C. Aelius Staienus, un autre homme nouveau, quod quia multis gratum erat et probabatur, [35] ascendisset ad honores ${ }^{83}$, si lui-même n'avait pas été condamné. À l'inverse enfin, Cicéron soulignait bien, à propos de Q. Aelius Tubero, comment la faiblesse du talent oratoire pouvait coûter sa carrière à un homme que sa naissance destinait aux plus hautes magistratures : sed ut uita sic oratione durus incultus horridus; itaque honoribus maiorum respondere non potuit ${ }^{84}$.

Ainsi, la compétition pour la supériorité oratoire ne se distinguait-elle pas des autres formes de compétition aristocratique pour la suprématie politique. La dignitas acquise par l'éloquence ne se démêlait pas de celle qu'apportait la gestion des magistratures. La concurrence ne trouvait à s'exercer que dans un champ unique, celui du dévouement à la cité et de l'exercice des honneurs. Voici comment Cicéron décrivait la situation de concurrence qui l'avait opposé à Hortensius :

\begin{abstract}
Cum igitur essem in plurumis causis et in principibus patronis quinquennium fere uersatus, tum in patrocinio Siciliensi maxume in certamen ueni designatus aedilis cum designato consule Hortensio ${ }^{85}$.

Après que j'eus, pendant cinq ans à peu près, plaidé un très grand nombre de causes et pris place parmi les premiers avocats, survint l'affaire des Siciliens, dont j'eus à défendre les intérêts, et ce fut là surtout que j'entrai en lutte avec Hortensius ; j'étais édile désigné et lui consul désigné.
\end{abstract}

L'éloquence en effet n'était que l'une de ces vertus qui permettaient de consacrer ses forces à ses concitoyens et qui justifiaient que l'on pût les diriger. Le seul critère qui permettait d'en apprécier la qualité était son efficacité et les seuls juges qui pouvaient en

\footnotetext{
${ }^{82}$ Brut.. 237.

${ }^{83}$ Brut.. 241. Ces deux personnages étaient plus exactement des senatores noui (cf. Wiseman 1971, $\mathrm{n}^{\circ} 448$, p. 267 et $n^{\circ} 410$, p. 262 ).

${ }^{84}$ Brut. 117. Il échoua à la préture de 128 . Son père $\left(R E \mathrm{I}, 1\right.$, col. $\left.535, \mathrm{n}^{\circ} 154\right)$ avait été le légat de Paul-Émile et avait épousé sa fille. Il était donc à la fois le petit-fils de Paul-Émile et le neveu de Scipion Emilien, cf. RE I, 1, col. 535-537, $\mathrm{n}^{\circ} 155$.

${ }^{85}$ Brut. 319 ; trad. J. Martha.
} 
décider étaient les citoyens qui bénéficiaient de ses effets. Elle s'inscrivait au cœur de l'action civique au même titre que la compétence juridique et la vertu militaire. Pour Cicéron, il n'était pas question de l'en séparer.

On comprend mieux ainsi ce qui fondait l'organisation par aetates qui déterminait le déroulement de tout le dialogue. La génération était précisément le cadre où s'imposait la compétition politique. C'était particulièrement vrai pour ceux qui avaient à peu près le même âge et qui avaient à lutter les uns [36] contre les autres pour l'accès aux magistratures. Mais c'était vrai aussi pour tous les contemporains pris dans un sens un peu large qui se trouvaient en concurrence d'autorité dans toutes les circonstances où leur avis comptait: devant le peuple, devant les tribunaux et surtout au Sénat où il fallait bien que le rang que l'on occupait fût conforté par l'assentiment que recevaient les opinions que l'on émettait.

On comprend surtout le sens que prenait dans le Brutus la polémique contre les Atticistes. Le reproche principal que Cicéron faisait à Licinius Calvus était précisément de ne pas capter le goût du public et surtout de ne pas le rechercher :

\begin{abstract}
Itaque eius oratio nimia religione attenuata doctis et attente audientibus erat inlustris, $<a>$ multitudine autem et a foro, cui nata eloquentia est, deuorabatur ${ }^{86}$.

Aussi son éloquence, trop réduite par cet excès de scrupule, était brillante pour des connaisseurs et des auditeurs attentifs ; mais sur la foule et le forum, pour qui l'éloquence est faite, elle passait sans être goûtée.
\end{abstract}

L'orateur devait puiser son succès auprès de la foule des auditeurs qui étaient en même temps des citoyens ${ }^{87}$. Se réfugier comme le faisait Licinius Calvus dans le jugement des spécialistes revenait d'une certaine manière à déserter la compétition et le combat politiques qui constituaient le champ d'action du véritable orateur ${ }^{88}$.

Il est vrai que l'époque y incitait. Le contrôle que César s'était gagné sur la vie politique enlevait de sa nécessité au jugement du peuple puisque désormais, c'était le vainqueur de la guerre civile qui décidait des compétences et arbitrait les concurrences. Comme il n'était plus autant indispensable de se faire reconnaître des autres citoyens, la compétition publique pour les honneurs perdait de son intérêt et entraînait dans son déclin la liberté aristocratique. Voilà pourquoi Cicéron, dans les dernières lignes qui nous sont restées du dialogue, incitait Brutus à ne pas abandonner l'espace public et à faire en sorte que lui-même l'emportât sur ses contemporains. Il devait conserver sa puissance à une éloquence active et tournée vers le peuple qui fût, comme dans les générations précédentes, le témoignage de la vitalité de la République et son lieu [37] d'expression ${ }^{89}$. On a beaucoup discuté du sens politique qu'il fallait donner à ce dialogue ${ }^{90}$. Sans doute trop. La leçon la plus évidente qu'il délivrait n'était ni de s'opposer vigoureusement à César ${ }^{91}$, ni d'appeler ce dernier à la composition ${ }^{92}$. Elle

\footnotetext{
${ }^{86}$ Brut. 283 ; trad. J. Martha modifiée.

${ }^{87}$ Brut. 289-290.

${ }^{88}$ Parmi les interprétations que l'on a données de cette querelle, cf. en part. Bringmann 1971, p. 24-40 ; Narducci 1997, p. 124-133.

${ }^{89}$ Brut. 331-333. Cf. en part. 331 : tuum enim forum, tuum illud curriculum, tu illuc ueneras unus [...]. Sur la relation entre les deux hommes, cf. l'étude de Rathofer 1986. Dugan 2005, p. 233-247, a bien montré que Cicéron réagissait à la tentation du retrait que Brutus exprimait sans doute dans son De uirtute.

${ }^{90}$ Cf. le bilan que fait Narducci 1997, p. 99-101.

${ }^{91}$ Cf. notamment Douglas 1966, p. 233.
} 
consistait d'abord à réaffirmer l'importance de l'éloquence comme cet instrument de compétition entre aristocrates ${ }^{93}$ qui leur permettait tout à la fois d'affirmer leur valeur et de se distinguer par l'adhésion qu'ils recevaient des autres citoyens.

Ainsi se résout aussi le paradoxe que je relevais en introduction. Pourquoi avoir fait apparaître la progression de l'éloquence romaine, si elle devait s'achever par un déclin ? Soumis comme tous les autres à la domination de César, Cicéron ne se contentait pas de regretter la crise où se trouvait la cité. Il montrait comment, de génération en génération, la compétition oratoire avait permis aux plus grands orateurs de se dégager de la foule des hommes politiques par la reconnaissance qu'ils obtenaient du peuple romain. Cette succession de grands hommes avait permis de construire la grandeur de Rome. Il ne s'agissait pas ici de conquêtes militaires, mais de cette maîtrise de la parole publique qui était l'une des conditions de la vie et de la paix communes. Chacun d'entre eux avait fait progresser le prestige de la cité avec la beauté et la puissance de l'éloquence. Ce n'était pas tant pour le goût de la littérature que parce que l'art de la parole était l'un des instruments qui permettait d'obtenir la reconnaissance du peuple. L'émulation pour la suprématie oratoire n'était qu'une forme particulière de la concurrence pour la domination politique. Certes, l'époque était difficile. Mais l'Histoire n'était pas close. Les plus jeunes devaient poursuivre l'effort, ne pas renoncer à s'investir dans l'espace public et tenir vivante cette manifestation profonde de la liberté aristocratique. [38]

Jean-Michel DAVID

Université Paris 1 Panthéon-Sorbonne

UMR 8210 ANHIMA

\footnotetext{
${ }^{92}$ Cf. Gelzer $1938^{1}$ et aussi sous certains aspects Bellincioni 1985, qui insiste cependant sur la réaffirmation du rôle de l'orateur.

${ }^{93}$ Cf. aussi les remarques de Steel 2002-2003, p. 210-211.
} 
Bibliographie

BECK H. et WALTER U. 2004, Die frühen römischen Historiker, II, Stuttgart.

BeLLincioni M. 1985, " Ancora sulle intenzioni politiche del "Brutus" », dans Sapienza antica, Studi in onore di Domenico Pesce, Milan, p. 49-67.

BLÖSEL W. 2000, « Die Geschichte des Begriffes mos maiorum von den Anfängen bis zu Cicero » in Linke B. und StEmmLER M. (eds), Mos maiorum, Untersuchungen zu den Formen der Identitätsstiftung und Stalilisierung in der römischen Republik, Stuttgart, Franz Steiner Verlag, p. 25-97.

Brigmann K. 1971, Untersuchungen zum späten Cicero, Hypomnemata 29, Göttingen.

Douglas A. E. 1966, M. Tulli Ciceronis Brutus, Oxford.

DugAn J. 2005, Making a New Man, Ciceronian self-fashioning in the rhetorical works, Oxford.

Flower H. I.1996, Ancestor Masks and Aristocratic Power in Roman Culture, Oxford, Clarendon Press.

Gelzer M. 1938, « Ciceros Brutus als politische Kundgebung », Philologus, 93, p. 128-131.

GILDENhARD I. 2003, "The 'Annalist' before the Annalists: Ennius and his Annales», in U. Eigler, U. Gotter, N. Luraghi, U. Walter éd., Formen römischer Geschichtsschreibung von den Anfängen bis Livius. Gattungen, Autoren, Kontexte, Darmstadt, Wissenschaftliche Buchgesellschaft, p. 93-114.

HÖLKESKAMP K.-J. 1996, « Exempla und Mos maiorum. Überlegungen zum kollektiven Gedächtnis der Nobilität » in GeHRKE H. J. und MülLER A. (eds), Vergangenheit und Lebenswelt. Soziale Kommunikation, Traditionsbildung und historisches Bewusstsein, Tübigen Gunter Narr Verlag, p. 301-338.

MENCACCI F. 2001, « Genealogia metaforica e maiores collettivi. Prospettive antropologiche sulla construzione dei viri illustres » in CoudRY M. et SPÄTH T. (eds), L'invention des grands hommes de la Rome antique, Die Konstruktion der grossen Männer Altroms, Paris, De Boccard, p. 421-437.

MutsChler F. H. 2000, « Norm und Erinnerung : Anmerkungen zur sozialen Funktion von historischem Epos und Geschichtsschreibung im 2. Jh. v. Chr. » in BRAUN N., Haltenhoff A, Mutschler F. H. (eds), Moribus antiquis res stat Romana, Römische Werte und römische Literatur im 3. und 2. Jh. V. Chr., München-Leipzig, K. G. Saur, p. 87-124.

NARDUCCI E. 1997, Cicerone e l'eloquenza romana, Retorica e progetto culturale, RomaBari.

NeuHauser W. 1958, Patronus und Orator. Eine Geschichte der Begriffe von ihren Anfängen bis in die augusteische Zeit, Innsbruck.

RATHOFER Cl. 1986, Ciceros Brutus als literarisches Paradigma eines Auctoritas Verhältnisses, Frankfurt/Main. 
RAWSON E. 1972, "Cicero the Historian and Cicero the Antiquarian », Journal of Roman Studies, 62, p. 33-45 (repris in RAWSON E. (ed.) 1991, Roman Culture and Society. Collected Papers, Oxford, Clarendon Press, p. 58-79).

RAWSON, E. 1985, Intellectual Life in the Late Roman Republic, Londres, Duckworth.

Schanz M. u. Hosius C. 1927, Geschichte der römischen Literatur bis zum

Gesetzgebungswerk des Kaisers Justinian, I, Die römische Literatur in der Zeit der Republik, München Beck.

Schanz M. u. Hosius C. 1935, Geschichte der römischen Literatur bis zum Gesetzgebungswerk des Kaisers Justinian, II, Die römische Literatur in der Zeit der Monarchie bis auf Hadrian, München Beck.

STEEL C. E. W 2002-2003, « Cicero's Brutus : the end of Oratory and the beginning of History? », BICS, 46, p. 195-211.

Sumner G. V. 1973, The Orators in Cicero's Brutus : Prosopography and Chronology, Phoenix supt. 11, Toronto.

VogT-SpIRA G. 2000, «Rednergeschichte als Literaturgeschichte. Ciceros Brutus und die Tradition der Rede in Rom » in C. NEUMEISTER ET W. RAECK (eds.) Rede und Redner, Bewertung und Darstellung in den antiken Kulturen, éd., Möhnesee, p. 207-225.

WALTER U. 2004, Memoria und res publica. Zur Geschichtskultur im republikanischen Rom, Frankfurt/Main, Antike.

Wiseman T. P. 1971, New Men in the Roman Senate, 139 B.C.-A.D. 14, Oxford.

ZANKer P.1987, Augustus und die Macht der Bilder, München, Beck. 